\title{
Beneficial Effects of Active Charge Balancing in Lithium-Ion Battery Systems
}

\author{
M. Raeber, A. Heinzelmann, and A. Taeschler
}

\begin{abstract}
This paper presents a theoretical analysis of the advantages of active charge balancing in lithium-ion battery systems and a comparison to passive balancing solutions.

Universal equations to estimate the benefits of an actively balanced battery system concerning energy savings and lifetime extension are derived from basic statistics. The calculations depend only on the configuration and aging properties of the individual cells. Their validity is limited to applications with full cycle usage and cells whose capacity values are normally distributed. The losses due to passive balancing in an $n \mathrm{SmP}$ battery system are calculated as well as the overall energy savings when applying active balancing. Furthermore, the extension factor for the lifetime of an actively balanced battery system is found to be in a range between 1.23 and 1.02 depending on the system configuration and the cell parameter. Based on the results, several options are identified to increase the energy efficiency of conventional passive balancing systems.
\end{abstract}

Index Terms-Active battery management system, active balancing, lithium-ion battery charging.

\section{INTRODUCTION}

For lithium-ion batteries, the use of an electronic battery management system (BMS) is essential for safety and operational reasons. It prevents cell failures caused by overcharging or deep discharging, monitors the temperature, and calculates the state of charge (SoC).

Individual cells in a stack of multiple cells in series tend to vary in capacity, which leads to a reduced battery capacity as weak cells reach charge and discharge limits earlier. As long as large battery systems consisted mainly of lead-acid or $\mathrm{NiCd} / \mathrm{NiMH}$ cells uneven charge distribution was a minor issue. Overcharging these cells led only to increased heat generation and charging losses. Lithium-ion cells, however, are much more sensitive to overvoltage. Therefore, a BMS with charge balancing function must be used to prevent the battery system from damage and premature battery failure. The balancer ensures that each cell is completely charged. Passive balancing is based on a resistive circuit to dissipate excess charge and thereby equalize the cells. Major drawbacks of passive balancing are a rather high balancing time due to the limited heat dissipation and a reduction in overall charging efficiency. Nowadays, as the demand for large lithium-ion based battery systems emerges, these drawbacks become more important to both battery system integrators and end-users.

That is when active BMS comes into play. Active

Manuscript received March 15, 2015; revised July 20, 2015.

The authors are with the Institute for Energy Systems and Fluid Engineering, School of Engineering, Zurich University of Applied Sciences, CH-8400 Winterthur, Switzerland (e-mail: manuel.raeber@zhaw.ch, andreas.heinzelmann@zhaw.ch, adrian.taeschler@zhaw.ch). balancing solutions for lithium-ion battery systems have been discussed extensively during the past few years. Further information and a comparison of different topologies are available in [1]-[5].

The main feature of active balancing is the efficient charge transfer from cells with high SoC to cells with low SoC. In Fig. 1 the basic process is shown for both charge and discharge process.

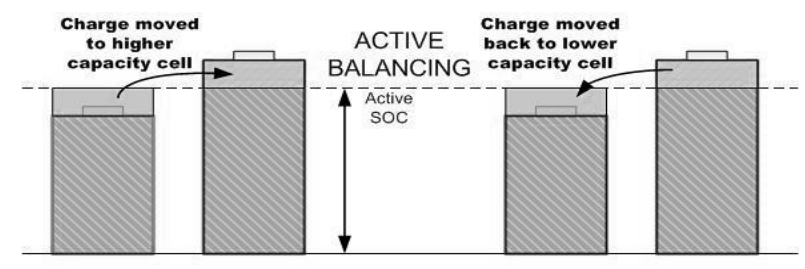

CHARGING

DISCHARGING

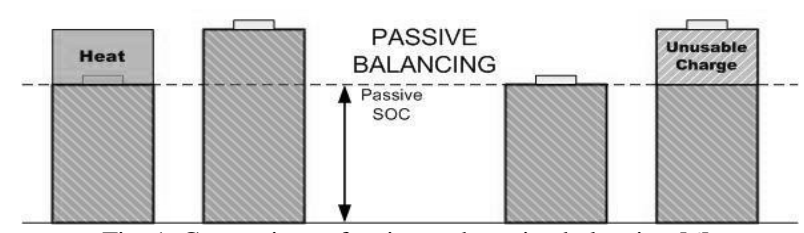

Fig. 1. Comparison of active and passive balancing [6]

\section{NOMENCLATURE}

The variables and symbols used in the calculations are defined in Table I.

TABLE I: MATHEMATICAL DEFINITIONS AND SYMBOLS

\begin{tabular}{ll}
\hline \hline Symbol & \\
\hline$a$ & Constant slope for linear capacity fade function \\
$b$ & Constant slope for linear standard deviation increase function \\
$c$ & Number of full cycles until EoL condition of battery system \\
$\eta$ & Efficiency of the active balancing circuit \\
$\mu$ & Arithmetic mean of cell capacities \\
$\sigma$ & Standard deviation of cell capacities \\
$n$ & Number of cells in series \\
$m$ & Number of cells in parallel \\
$C$ & Capacity \\
$Q$ & Charge \\
$W$ & Energy \\
$U$ & Voltage \\
\hline \hline
\end{tabular}

\section{FUNDAMENTALS OF PASSIVE BALANCING}

\section{A. Capacity Distribution}

It is assumed that the battery cell capacities are normally distributed acc. to the Gaussian density function with the variables $\mu=$ mean and $\sigma=$ standard deviation:

$$
f(x)=\frac{1}{\sqrt{2 \pi \sigma^{2}}} e^{\frac{(x-\mu)^{2}}{2 \sigma^{2}}}
$$


The mean and the expected value are identical for symmetric functions:

$$
E(x)=\mu=\int_{-\infty}^{+\infty} x f(x) d x
$$

The cumulative density function cannot be calculated analytically. It is defined as the integral of the density function and can be written as follows:

$$
F(x)=\int f(x) d x
$$

and

$$
F(x)=0.5\left[1+\operatorname{erf}\left(\frac{x-\mu}{\sqrt{2 \sigma}}\right)\right]
$$

The assumption about a normal distribution is supported by measurements published in [7]. Cells with a deviation of more than approximately $2.75 \sigma$ do not show up in the histogram in Fig. 2 since they fail the factory quality test and are not shipped.

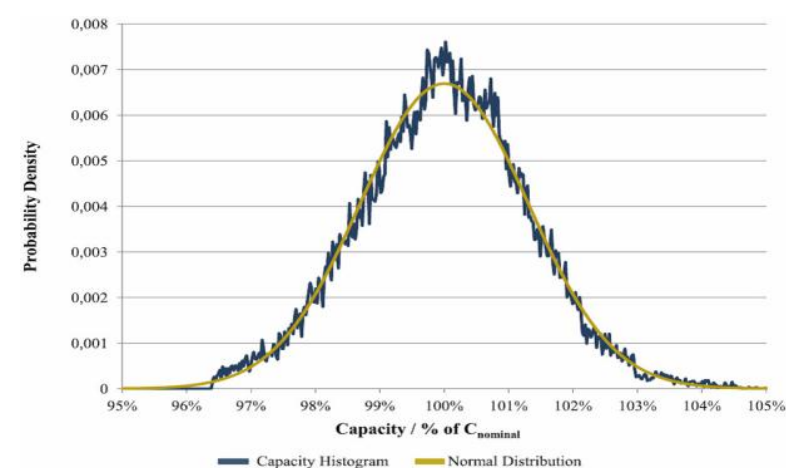

Fig. 2. Distribution of cell capacities at Beginning of Life (BoL) (20’000 measured cells, $\sigma=1.3 \%$ ) [7].

For a more realistic consideration, the density function of the battery cell capacities is not assumed exactly Gaussian but cut at the edges and rescaled to fulfill the equation in (5).

With $\mu$ and $\sigma$ being the mean value and the standard deviation of the deployed cell, it is assumed that the capacity range of all cells is within $\mu \pm 2 \sigma$.

The integral of the density function is hence defined as

$$
\int_{-\infty}^{+\infty} f(x) d x=\int_{-\infty}^{+\infty} f^{*}(x) d x=\int_{\mu-2 \sigma}^{\mu+2 \sigma} f^{*}(x) d x=1
$$

The two density functions for $\mu=10 \mathrm{Ah}$ and $\sigma=0.15 \mathrm{Ah}$ and margins at $\mu \pm 2 \sigma$ are shown in Fig. 3 .

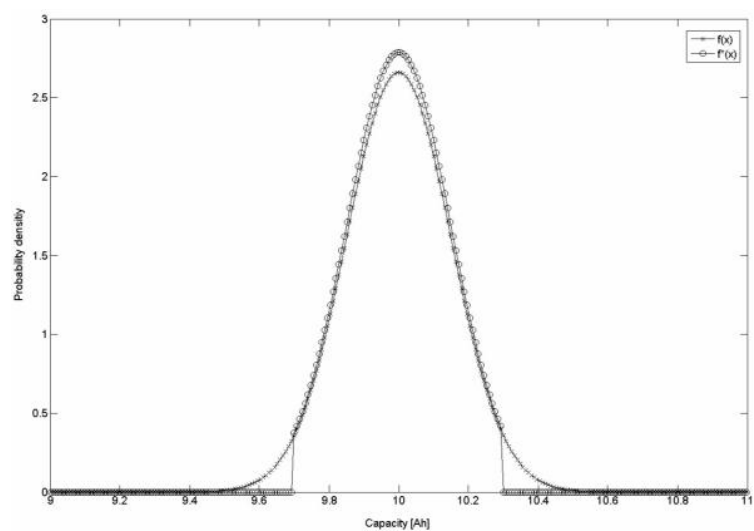

Fig. 3. Gaussian and adjusted density functions $f(x)$ and $f^{*}(x)$.

\section{B. nS1P Battery Systems}

During passive balancing, charge is dissipated from cells that are close to their maximum charging voltage. This process lasts until the strongest cell has reached its maximum charging voltage. For a battery system with an $n \mathrm{~S} 1 \mathrm{P}$ configuration ( $n$ cells in series, 1 cell in parallel), the charge difference of a cell with a capacity of $x$ Ah in relation the strongest cell is given as:

$$
\Delta Q=\mu+2 \sigma-x
$$

The total balanced charge of all cells can be calculated as follows:

$$
Q_{\text {Bal_pack }}=n \int_{\mu-2 \sigma}^{\mu+2 \sigma}(\mu+2 \sigma-x) f^{*}(x) d x
$$

Using (2) and (5), (7) can be simplified to

$$
Q_{\text {Bal_pack }}=2 n \sigma \text {. }
$$

The energy losses during charging due to passive balancing sum up to

$$
W_{\text {Passive }}=U_{B a l_{\_} a v g} Q_{\text {Bal_pack }}=2 n \sigma U_{\text {Bal_avg }}
$$

$U_{\text {Bal_avg }}$ is the average voltage of the affected cell during the balancing process. For lithium-ion cells, in most applications $U_{\text {Bal_avg }}$ will be between 4.0 and $4.1 \mathrm{~V}$.

\section{C. nSmP Battery Systems}

For battery systems with more than one cell on each stack level ( $n \mathrm{~S} m \mathrm{P})$, the calculation has to be adjusted.

According to probability theory [8] the resulting mean and standard deviation for independent random variables are $\mu^{\prime}=m \mu$ and $\sigma^{\prime}=\sqrt{m} \sigma$. Using $\mu^{\prime}$ and $\sigma^{\prime}$ instead of $\mu$ and $\sigma$ in (1), the density function becomes

$$
f_{m}(x)=\frac{1}{\sqrt{2 \pi \sigma^{\prime 2}}} e^{\frac{\left(x-\mu^{\prime}\right)^{2}}{2 \sigma^{\prime 2}}}
$$

In line with (6), the total balanced charge can be written as

$$
Q_{m_{\text {Bal_pack }}}=n \int_{\mu \prime-2 \sigma^{\prime}}^{\mu \prime+2 \sigma^{\prime}}\left(\mu^{\prime}+2 \sigma^{\prime}-x\right) f_{m}^{*}(x) d x \text {. }
$$

Using (2) and (5), (11) can be simplified to

$$
Q_{m_{\text {Bal_pack }}}=2 n \sigma^{\prime}=2 n \sqrt{m} \sigma .
$$

The energy losses during charging due to passive balancing sum up to

$$
W_{m_{\text {Passive }}}=U_{B a l_{\_} a v g} Q_{m_{B a l \_p a c k}}=2 n \sqrt{m} \sigma U_{\text {Bal_avg }}
$$

\section{BENEFITS OF ACTIVE BALANCING}

\section{A. Energy Savings}

If active balancing is used instead of passive balancing, most of the equalizing losses can be avoided. An overall 
energy efficiency of up to $77 \%$ has been shown for an active balancing prototype in [9]. Given an energy efficiency of $\eta$, it follows that the energy losses during charging due to active balancing are

$$
W_{m_{\text {Active }}}=(1-\eta) 2 n \sqrt{m} \sigma U_{\text {Bal_avg. }} .
$$

The balancing effort of a battery system increases with its lifetime. Varying operation conditions lead to inhomogeneous aging of individual cells. While the mean of a battery pack's capacity decreases, the standard deviation of the cells increases. In [7] the standard deviation had almost doubled after the End-of-life (EoL) condition of $80 \%$ remaining SoC was reached. The aging behavior of lithium-ion cells is depending on operation conditions such as the load profile, the charging current and cell temperatures. Characteristic curves can be found in [10]-[13] or [14]. For a simple consideration, a linear aging function of both $\mu$ and $\sigma$ is assumed. Hence, the energy savings during the battery's lifetime due to active balancing sum up to

$$
W_{t o t}=\text { cycles } * 2 \eta n \sqrt{m} \frac{\sigma_{B o L}+\sigma_{E o L}}{2} U_{B a l_{\_} a v g} .
$$

\section{B. Capacity Increase}

The lifetime of a battery pack is usually defined as a minimum remaining capacity in relation to the initial capacity (usually between 70 and 80 percent). The weakest cell, or cell level in case of more than one parallel cell, in a battery system determines the total capacity which is

$$
C_{\text {Pack }}=\mu^{\prime}-2 \sigma^{\prime}=m \mu-2 \sqrt{m} \sigma .
$$

If active balancing is performed during discharge as well, the useable capacity can be increased. For an actively balanced battery pack this capacitance value depends on the efficiency and power of the balancing electronics and can get as high as

$$
\begin{gathered}
C_{\text {Pack_active }}=\mu^{\prime}-(1-\eta) 2 \sigma^{\prime}= \\
m \mu-(1-\eta) 2 \sqrt{m} \sigma .
\end{gathered}
$$

Fig. 4 and Fig. 5 show the capacity fade during aging of a passive respectively active balancing system for different configurations.

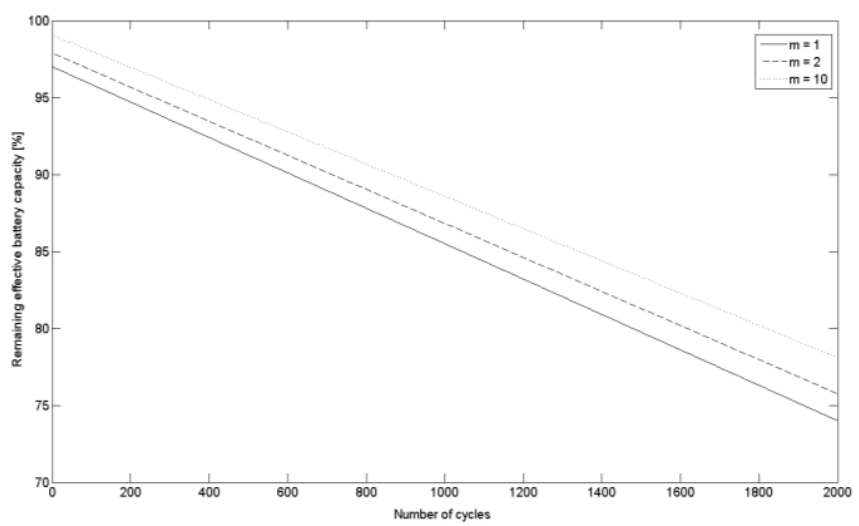

Fig. 4. Effective capacity of a passive balancing battery system with 1, 2 and 10 parallel cells over lifetime acc. to $(16)\left(\sigma_{\mathrm{BoL}}=1.5 \%, a=-10^{-4}\right.$ and $b=$ $\left.5 \times 10^{-4}\right)$.

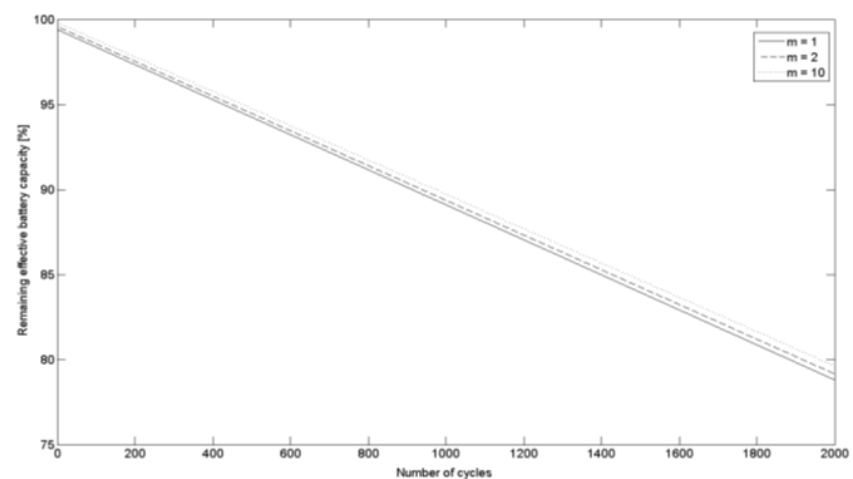

Fig. 5. Effective capacity of an active balancing battery system with 1,2 and 10 parallel cells over lifetime according to (17) $\left(\sigma_{\mathrm{BoL}}=1.5 \%, a=-10^{-4}, b=\right.$ $5 \times 10^{-4}$ and $\left.\eta=0.8\right)$.

\section{Lifetime Extension}

$x$ is defined as the number of full cycles and $\mu(0)=\mu_{B o L}$, $\sigma(0)=\sigma_{B o L}$. It is assumed that the aging functions of $\mu$ and $\sigma$ are linear over the number of cycles. Hence,

$$
\begin{gathered}
\mu^{\prime}(x)=m \mu(x)=m(1+a x) \mu_{B o L}, \\
\sigma^{\prime}(x)=\sqrt{m} \sigma(x)=\sqrt{m}(1+b x) \sigma_{B o L}, \\
k=\frac{\text { cycles }_{\text {act }}}{\text { cyclespass }_{\text {pand }}} \text {, and }=\text { cycles }_{\text {pass }} .
\end{gathered}
$$

In (16) and (17) it has been shown that the effective capacity of a battery system is different for passive and active balancing systems. By setting equal (16) and (17) and using the following definitions, the lifetime extension factor $k$ can be calculated in (18):

$$
\begin{gathered}
\mu_{E o L_{-} p}^{\prime}=\mu^{\prime}(c), \sigma_{E o L_{-} p}^{\prime}=\sigma^{\prime}(c), \\
\mu_{E O L_{-} a}^{\prime}=\mu^{\prime}(k c), \sigma_{E o L_{-} a}^{\prime}=\sigma^{\prime}(k c), \\
k=\frac{a \sqrt{m} \mu_{B o L+2\left(\frac{1-\eta}{c}-b\right) \sigma_{B o L}}}{a \sqrt{m} \mu_{B o L^{-}}-2 b(1-\eta) \sigma_{B o L}} .
\end{gathered}
$$

For $c \gg 1,(18)$ becomes independent of $c$ :

$$
k \approx \frac{a \sqrt{m} \mu_{B o L}-2 b \sigma_{B o L}}{a \sqrt{m} \mu_{B o L}-2 b(1-\eta) \sigma_{B o L}} .
$$

The extension factor $k$ is shown in Fig. 6 for an exemplary battery system with varying $m$ and two different cell capacity standard deviations.

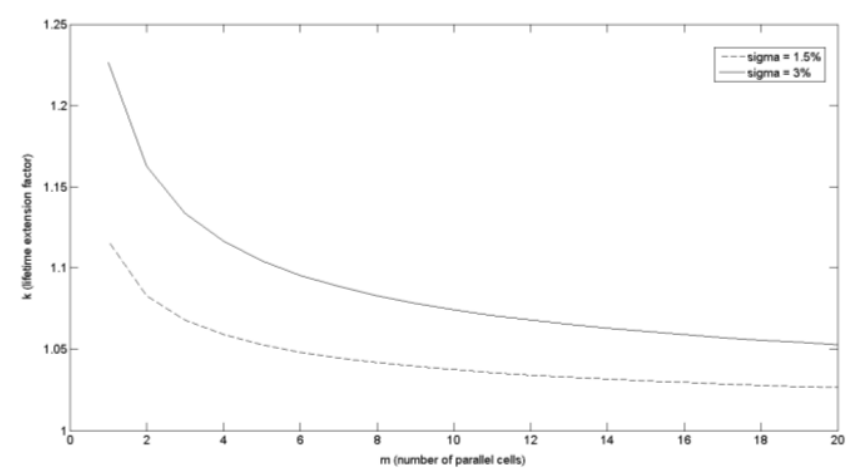

Fig. 6. Lifetime extension factor due to active balancing of a battery system with m parallel cells acc. to (19) $\left(\sigma_{\mathrm{BoL}}=1.5 \%-3 \%, a=-10^{-4}, b=5 \times 10^{-4}, \eta=0.8\right)$. 


\section{CONCLUSION}

It has been shown that the theoretical advantages of active charge balancing in battery systems can be calculated analytically with knowledge of just few cell parameters and the efficiency factor of the active balancing stage. The lifetime extension factor due to active balancing can be estimated independently of the cycle number. Considering the obtained equations, several ways to increase the energy efficiency of passively balanced battery systems can be derived:

1) Equation (9) and (13) show that the balancing losses are reduced when using more than one cell per level in a battery system ( $n \mathrm{~S} m \mathrm{P}$ instead of $n \mathrm{~S} 1 \mathrm{P})$.

2) Avoiding full cycles reduces the balancing effort.

3) Presorted cells have a lower $\sigma$ and lead to fewer balancing losses according to (9) and (13).

In case none of these suggestions can be applied to a given battery system, active balancing might be a good approach to reduce losses and increase energy efficiency. A further reduction in balancing losses can be achieved if the balancing is performed during the charging process at a lower cell voltage regarding (14).

Further investigations should be made for battery systems that are not operated only in full cycles. The assumption that mean and standard deviation are linear functions over cycle numbers is a simplification. If future measurements show a different behavior, the affected equations must be updated.

\section{REFERENCES}

[1] J. Gallardo-lozano, E. Romero-cadaval, M. I. Milanes-montero, and M. A. Guerrero-martinez, "Battery equalization active methods," Journal of Power Sources, vol. 246, pp. 934-949, 2014.

[2] F. Baronti, R. Roncella and R. Saletti, "Performance comparison of active balancing techniques for lithium-ion batteries," Journal of Power Sources, vol. 267, pp. 603-609, Dec. 2014.

[3] Y. Lee and M. Cheng, "Intelligent Control Battery Equalization for Series Connected Lithium-Ion Battery Strings," IEEE Transactions on Industrial Electronics, vol. 52, no. 5, pp. 1297-1307, 2005.
[4] "Active Charge Balancing for Li-ion Battery Stacks," Power Electronics Europe, no. 3, pp. 25-27, 2008.

[5] D. D. Quinn and T. T. Hartley, "Design of novel charge balancing networks in battery packs," Journal of Power Sources, vol. 240, pp. 26-32, Oct. 2013.

[6] J. Marcinkowski, "Large Li-ion battery packs: Active balancing improves many parameters," EE Times Europe, pp. 1-3, 2014.

[7] S. Paul, C. Diegelmann, H. Kabza and W. Tillmetz, "Analysis of ageing inhomogeneities in lithium-ion battery systems," Journal of Power Sources, vol. 239, pp. 642-650, Oct. 2013.

[8] L. Meier, Statistik und Wahrscheinlichkeitsrechnung, ETH Zürich, 2015 .

[9] A. Ramirez and B. Miesch, "Semester Project ( ET12b ): Active balancing battery management system for up to $8 \mathrm{Li}$-Ion cells," Zurich University of Applied Sciences, 2014.

[10] A. T. Stamps, C. E. Holland, R. E. White and E. P. Gatzke, "Analysis of capacity fade in a lithium ion battery," Journal of Power Sources, vol. 150 , pp. 229-239, Oct. 2005.

[11] N. Omar, M. A. Monem, Y. Firouz, J. Salminen, J. Smekens, O. Hegazy, H. Gaulous, G. Mulder, P. Van den Bossche, T. Coosemans and J. Van Mierlo, "Lithium iron phosphate based battery Assessment of the aging parameters and development of cycle life model," Applied Energy, vol. 113, pp. 1575-1585, Jan. 2014.

[12] G. Sarre, P. Blanchard and M. Broussely, "Aging of lithium-ion batteries," Journal of Power Sources, vol. 127, no. 1-2, pp. 65-71, Mar. 2004.

[13] G. Ning, B. Haran and B. N. Popov, "Capacity fade study of lithium-ion batteries cycled at high discharge rates," Journal of Power Sources, vol. 117, no. 1-2, pp. 160-169, May 2003.

[14] P. Rong, S. Member and M. Pedram, "An Analytical Model for Predicting the Remaining Battery Capacity of Lithium-Ion Batteries," IEEE Transactions on Very Large Scale Integration Systems, vol. 14, no. 5, pp. 441-451, 2006.

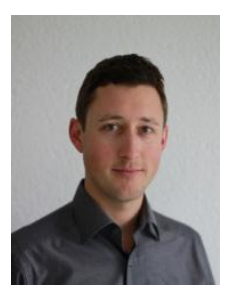

Manuel Raeber has received his master degree in electrical engineering and information technology from the Swiss Federal Institute of Technology (ETH) in Zurich, Switzerland in 2008.

He currently works as a research associate within the Institute of Energy Systems and Fluid Engineering (IEFE) at the Zurich University of Applied Sciences (ZHAW) in Winterthur, Switzerland. His field of research covers power electronic converters for the renewable energy, active battery management and lifetime behavior of battery cells and systems. 